\title{
Stationary solution of plane SH-wave scattered by semi-cylindrical canyon in a quarter space

\author{
Hui Qi ${ }^{1, a}$, Chun Gao ${ }^{1,2, b^{*}}$, Xiang-Nan Pan ${ }^{1, c}$
}

${ }^{1}$ College of Aerospace and Civil Engineering, Harbin Engineering University, Harbin 150001, China

${ }^{2}$ Department of Civil Engineering, Harbin University, Harbin 150086, China

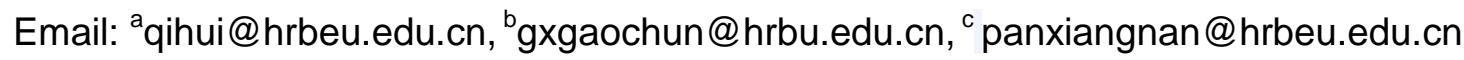

Keywords: SH-wave, semi-cylindrical canyon, quarter space, stationary solution, scattering wave. Abstract: According to wave function expansion method and image method, the anti-plane steady state response of a plane $\mathrm{SH}$-wave scattered by a semi-cylindrical canyon in a quarter space is analyzed, and the specific form of displacement field and stress field of the elastic wave are obtained. According to the convergence of wave function series, the near field solution is analyzed, and the far field solution of scattering wave by cylindrical canyon is obtained through asymptotic behavior of Hankel function and Graf addition formula. The results show that the wave number, incidence angle and the distance between canyon and vertical boundary greatly affect the stationary solution of SH wave scattering.

\section{Introduction}

The scattering of elastic waves by local site conditions has been concerned by mathematical physics and mechanics scientists, the researchers have made lots of achievements [1-4]. In recent years, Liu D. K. and others [5,6] studied the far field solution of plane SH-wave by interface circular hole, and the scattering of SH-waves by heave and cavity in half space. Liang J. W. and others [7,8] studied the scattering of plane $\mathrm{SH}$ waves by a circular-arc layered canyon in a half space and wedge-shaped space. Chen T. Y. [9] studied the dynamic response of upright incidence of $\mathrm{SH}$-waves at semi-cylindrical interface with a circular lining structure. Qi H. and others [10-15] studied the anti-plane cylindrical scattering of steady SH-waves by cavity, inclusion, crack and heave in half-space and quarter space; the dynamic stress concentration factor and surface displacement amplitude are calculated. However, most of these results are limited to the near field problems, few studied on the far field of the scattering wave. In this paper, according to the wave function expansion method and image method, the anti-plane response of plane SH-wave by semi-cylindrical canyon in quarter field is analyzed; it not only solved the dynamic stress concentration factor of near field, but also analyzed the far field displacement mode. In this way, the analysis method and results of this paper have certain value in theoretical research and engineering application.

\section{Description and Analysis}

SH-wave is the simplest elastic wave, its vibration vector and propagation vector are perpendicular to each other. As a two-dimensional problem, the displacement and the material coordinate are decoupled, and satisfy the scalar wave equation (1). In the equation, $\mu$ and $\rho$ are the shear modulus and mass density of the elastic medium, $\Delta$ is a two-dimensional Laplace operator. Deal with Eq. (1) by separation of variables, omitting the time harmonic function $\exp (-i \omega t)$, the Helmholtz equation (2) was established.

$$
\begin{aligned}
& \mu \Delta w=\rho \ddot{w} \\
& \Delta w+k^{2} w=0, k=\omega \sqrt{\frac{\rho}{\mu}}
\end{aligned}
$$


The elastodynamic boundary value in this paper is shown in Fig. 1. There are two stress-free planes in the propagation plane of $\mathrm{SH}$-wave in the quarter space, the horizontal boundary is plane $B_{H}$, and the vertical is plane $B_{V}$. There is a semi- cylindrical canyon which radius is $R$ in the horizontal boundary plane $B_{H}$, the center point is $O$, and the direct distance between $O$ and vertical boundary plane $B_{V}$ is $d$; the boundary of the semi- cylindrical canyon $B_{C}$ is a stress-free cylindrical surface. Build a plane rectangular coordinate $\operatorname{system}(O, x, y)$, let the center point $O$ of semicylindrical canyon as origin, normal vector direction of vertical boundary plane $B_{V}$ as positive direction of axis $x$, normal vector direction of horizontal boundary plane $B_{H}$ as positive direction of axis $y$. and out-plane direction as positive direction of axis $z$. Thus, the anti plane displacement $w$ is a function of $x$ and $y$ in the direction of axis $z$. Set steady plane SH-wave, the expression of the anti plane displacement $w_{i}^{1}$ is Eq. (3). $w_{i}^{1}(x, y)$ is the displacement field which the time harmonic function $\exp (-i \omega t)$ is omitted, as shown in Eq. (4). $w$ is the time amplitude of vibration vector of SH-wave, that is anti plane amplitude. $\alpha$ is the angle between propagation vector and axis $x$, it means incident angle.

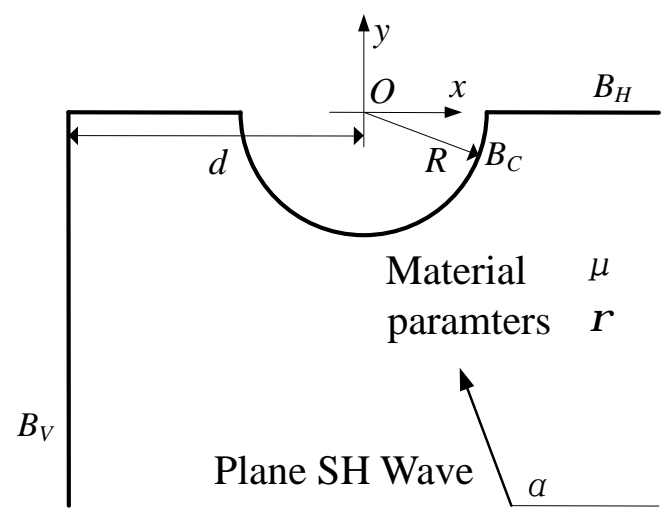

Fig. 1 Model description

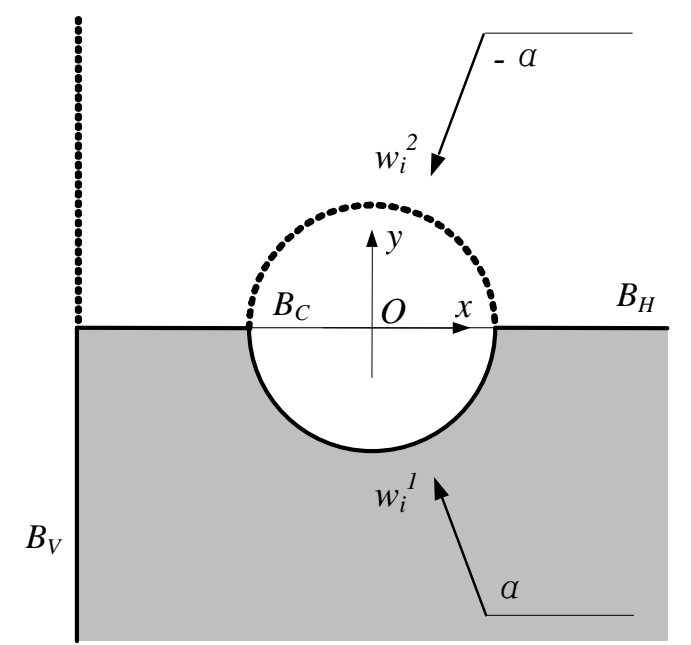

Fig. 2 Extended canyon

$$
\begin{aligned}
& w_{i}^{1}=w_{i}^{1}(x, y) \exp (-i \omega t) . \\
& w_{i}^{1}(x, y)=W \exp [i k(x \cos \alpha+y \sin \alpha)] .
\end{aligned}
$$

Considering the stress free condition on the horizontal boundary, let plane $B_{H}$ as the symmetry plane, an equivalent boundary value problem is established. As shown in Fig. 2. The semi-circular boundary $B_{C}$ is extended as a circle, and the quarter space is extended as a half space. As a result, the semi-cylindrical canyon in the quarter space is extended as a cylindrical canyon in a vertical half space. Similarly, the incident wave $w_{i}^{1}$ is extended to $w_{i}^{1}+w_{i}^{2}$, the displacement field $w_{i}^{2}(x, y)$ is defined as equation (5)

$$
w_{i}^{2}(x, y)=W \exp [i k(x \cos \alpha-y \sin \alpha)]
$$

In this paper, the time harmonic function $\exp (-i \omega t)$ was omitted. Thus, as an equivalent problem, the pan-setting equation of displacement $w$ is governing equation (2), the determining solution is the stress free condition of the vertical boundary plane $B_{V}$ and the cylindrical boundary $B_{C}$.

\section{Definite solution problem}

\section{Form of displacement field.}

Introducing complex variable $z$ and conjugate complex variable $\bar{z}$, according to equation (6), polar coordinate system $(O, r, \theta)$ is defined. Combining the wave function expansion and the mirror 
image method, the displacement field equation (7) of the cylindrical scattering wave $w_{s}$ is obtained. In the equation, $A_{n}$ is the undetermined wave function coefficient, $z^{\prime}=z+2 d, D$ and $D^{\prime}$ are intermediate variables defined by Eq. (8), $H_{n}^{(1)}$ is the first kind of the Hankel function of $n$ order.

$$
\begin{aligned}
& \left\{\begin{array}{l}
z=x+i y=r e^{i \theta} \\
\bar{z}=x-i y=r e^{-i \theta}
\end{array}\right. \\
& w_{s}(z)=\sum_{n=-\infty}^{+\infty} A_{n}\left[D(z)+D^{\prime}\left(z^{\prime}\right)\right] \\
& \left\{\begin{array}{l}
D(z)=H_{n}^{(1)}(k|z|)\left(\frac{z}{|z|}\right)^{n} \\
D^{\prime}\left(z^{\prime}\right)=(-1)^{n} H_{n}^{(1)}\left(k\left|z^{\prime}\right|\right)\left(\frac{z^{\prime}}{\left|z^{\prime}\right|}\right)^{-n}
\end{array}\right.
\end{aligned}
$$

According to the stress equation of inclined plane, the radial stress component $\tau_{r z}^{s}$ and the circumferential stress component $\tau_{\theta z}^{s}$ of the scattering wave by the cylindrical canyon are obtained, shown as Eq. (9). In the formula, all the intermediate variables are defined by Eq. (10) - (13); $\Delta \theta=\theta-\theta^{\prime}, \theta^{\prime}=\arg z^{\prime}$ are the argument principal value of $z^{\prime}$.

$$
\begin{aligned}
& \left\{\begin{array}{l}
\tau_{r z}^{s}(z)=\sum_{n=-\infty}^{+\infty} A_{n}\left[S_{r}(z)+S_{r}{ }^{\prime}\left(z^{\prime}\right)\right] \\
\tau_{\theta z}^{s}(z)=\sum_{n=-\infty}^{+\infty} A_{n}\left[S_{\theta}(z)+S_{\theta}{ }^{\prime}\left(z^{\prime}\right)\right]
\end{array}\right. \\
& \left\{\begin{array}{l}
S_{r}(z)=\frac{k \mu}{2} H_{-}(z)\left(\frac{z}{|z|}\right)^{n} \\
S_{\theta}(z)=\frac{i k \mu}{2} H_{+}(z)\left(\frac{z}{|z|}\right)^{n}
\end{array}\right. \\
& \left\{\begin{array}{l}
S_{r^{\prime}}{ }^{\prime}\left(z^{\prime}\right)=S_{r^{\prime}}\left(z^{\prime}\right) \cos (\Delta \theta)+S_{\theta^{\prime}}\left(z^{\prime}\right) \sin (\Delta \theta) \\
S_{\theta}{ }^{\prime}\left(z^{\prime}\right)=-S_{r^{\prime}}\left(z^{\prime}\right) \sin (\Delta \theta)+S_{\theta^{\prime}}\left(z^{\prime}\right) \cos (\Delta \theta) \\
S_{r^{\prime}}\left(z^{\prime}\right)=(-1)^{n} \frac{k \mu}{2} H_{-}\left(z^{\prime}\right)\left(\frac{z^{\prime}}{\left|z^{\prime}\right|}\right)^{-n} \\
S_{\theta^{\prime}}\left(z^{\prime}\right)=(-1)^{n+1} \frac{i k \mu}{2} H_{+}\left(z^{\prime}\right)\left(\frac{z^{\prime}}{\left|z^{\prime}\right|}\right)^{-n}
\end{array}\right. \\
& \left\{\begin{array}{l}
H_{-}(\bullet)=H_{n-1}^{(1)}(k|\bullet|)-H_{n+1}^{(1)}(k|\bullet|) \\
H_{+}(\bullet)=H_{n-1}^{(1)}(k|\bullet|)+H_{n+1}^{(1)}(k|\bullet|)
\end{array}\right.
\end{aligned}
$$

In addition to the scattering wave of the cylinder, the extended incident wave $w_{i}^{1}+w_{i}^{2}$ is reflected by the vertical boundary plane $B_{V}$, and produce the reflected wave $w_{r}^{1}+w_{r}^{2}$, the displacement fields are given by Eq. (14), $x^{\prime}$ and $y^{\prime}$ are real part and imaginary part respectively. Similarly, according to the inclined plane stress equation, the Eq. (15) is obtained. $\tau_{r z}^{p}$ and $\tau_{\theta z}^{p}$ are the radial and circumferential stress component respectively. In the formula, all of the intermediate variables are defined by Eq. (16) - (19) 


$$
\begin{aligned}
& \left\{\begin{array}{l}
w_{r}^{1}\left(x^{\prime}, y^{\prime}\right)=W \exp \left[i k\left(-x^{\prime} \cos \alpha+y^{\prime} \sin \alpha\right)\right] \\
w_{r}^{2}\left(x^{\prime}, y^{\prime}\right)=W \exp \left[i k\left(-x^{\prime} \cos \alpha-y^{\prime} \sin \alpha\right)\right]
\end{array}\right. \\
& \left\{\begin{array}{l}
\tau_{r z}^{p}(z)=P_{r}(z)+P_{r}{ }^{\prime}\left(z^{\prime}\right) \\
\tau_{\theta z}^{p}(z)=P_{\theta}(z)+P_{\theta}{ }^{\prime}\left(z^{\prime}\right)
\end{array}\right. \\
& \left\{\begin{array}{l}
P_{r}(z)=P_{x}(z) \cos \theta+P_{y}(z) \sin \theta \\
P_{\theta}(z)=-P_{x}(z) \sin \theta+P_{y}(z) \cos \theta
\end{array}\right. \\
& \left\{\begin{array}{l}
P_{r}{ }^{\prime}\left(z^{\prime}\right)=P_{x^{\prime}}\left(z^{\prime}\right) \cos \theta+P_{y^{\prime}}\left(z^{\prime}\right) \sin \theta \\
P_{\theta}{ }^{\prime}\left(z^{\prime}\right)=-P_{x^{\prime}}\left(z^{\prime}\right) \sin \theta+P_{y^{\prime}}\left(z^{\prime}\right) \cos \theta
\end{array}\right. \\
& \left\{\begin{array}{l}
P_{x}(z)=i k \mu \cos \alpha\left[w_{i}^{1}(x, y)+w_{i}^{2}(x, y)\right] \\
P_{y}(z)=i k \mu \sin \alpha\left[w_{i}^{1}(x, y)-w_{i}^{2}(x, y)\right]
\end{array}\right. \\
& \left\{\begin{array}{l}
P_{x^{\prime}}\left(z^{\prime}\right)=-i k \mu \cos \alpha\left[w_{r}^{1}\left(x^{\prime}, y^{\prime}\right)+w_{r}^{2}\left(x^{\prime}, y^{\prime}\right)\right] \\
P_{y^{\prime}}\left(z^{\prime}\right)=i k \mu \sin \alpha\left[w_{r}^{1}\left(x^{\prime}, y^{\prime}\right)-w_{r}^{2}\left(x^{\prime}, y^{\prime}\right)\right]
\end{array}\right.
\end{aligned}
$$

\section{Satisfied Boundary conditions.}

The displacement field of the cylindrical scattering wave $w_{s}$ is symmetric about the vertical boundary plane $B_{V}$, the stress field obviously satisfies the free condition in plane $B_{V}$. The reflected wave $w_{r}^{1}+w_{r}^{2}$ is generated by incident wave $w_{i}^{1}+w_{i}^{2}$ based on the stress free conditions in the plane $B_{V}$, their stress field is zero on the vertical boundary of the half space. Thus, there is only one boundary condition is not satisfied, that is the stress free condition in the cylindrical surface $B_{C}$ of the extended canyon, it is called the definite solution condition of scattering wave. By substituting Eq. (9) and (15), the control equation of scattering wave (20) is obtained. $|z|=R, \tau_{r z}^{p}(z)$ and $S_{r}(z)+S_{r}{ }^{\prime}\left(z^{\prime}\right)$ are functions of $\theta$.

This is a functional equation about the argument $\theta$. In order to solve the infinite number of undetermined wave function coefficients $A_{n}$, through the Fourier expansion of Eq. (20), the series equation (21) is obtained. $F_{m}$ and $F_{m}^{n}$ are the Fourier coefficients defined by Eq. (22). The equivalent linear algebraic equation (23) are obtained by the orthogonality of the harmonic function $\exp (\operatorname{im} \theta)$.

$$
\begin{aligned}
& \tau_{r z}^{p}(z)+\sum_{n=-\infty}^{+\infty} A_{n}\left[S_{r}(z)+S_{r}{ }^{\prime}\left(z^{\prime}\right)\right]=0 \\
& \left(\sum_{m=-\infty}^{+\infty} F_{m}+\sum_{n=-\infty}^{+\infty} A_{n} \sum_{m=-\infty}^{+\infty} F_{m}^{n}\right) \exp (i m \theta)=0 \\
& \left\{\begin{array}{l}
F_{m}=\int_{0}^{2 \pi} \tau_{r z}^{p}(z) \exp (-i m \theta) d \theta \\
F_{m}^{n}=\int_{0}^{2 \pi}\left[S_{r}(z)+S_{r}{ }^{\prime}\left(z^{\prime}\right)\right] \exp (-i m \theta) d \theta
\end{array}\right.
\end{aligned}
$$




$$
\left\{\begin{array}{c}
F_{-\infty}+\sum_{n=-\infty}^{+\infty} A_{n} F_{-\infty}^{n}=0 \\
F_{m}+\sum_{n=-\infty}^{+\infty} A_{n} F_{m}^{n}=0 \\
F_{+\infty}^{+}+\sum_{n=-\infty}^{+\infty} A_{n} F_{+\infty}^{n}=0
\end{array}\right.
$$

\section{Steady solution}

\section{Convergence and near field solution.}

According to the linear algebraic equations (23), truncates wave function series (7), and the expressions for the cylindrical scattering wave $w_{s}$ can be obtained. It is necessary to consider the convergence of this expression to represent the displacement field of the scattering wave. On the canyon which is extended as a cylinder, according to the asymptotic behavior of the first kind of Hankel function at zero, Eq. (24), evaluates the convergence of series (7), $B_{n}^{1}$ and $B_{n}^{2}$ are constants that depend on order $n$. Set argument $\bullet=k R$, as order $n$ increase, the modulus of the Hankel function increases rapidly with the negative power exponent of the argument. As the Fourier coefficients $F_{m}$ of the incident and reflected waves are constant, the undetermined coefficient $A_{n}$ decreases rapidly with positive power exponent of the argument. In this way, the convergence of the wave function series (7) is proved, when the radius $R$ is constant, the smaller value $k$ is, the better the convergence could be.

$$
H_{n}^{(1)}(\bullet) \stackrel{\bullet \rightarrow 0}{\longrightarrow} B_{n}^{1}\left(\frac{\bullet}{2}\right)^{|n|}+i B_{n}^{2}\left(\frac{\bullet}{2}\right)^{-|n|}
$$

When the incident wave frequency is not too high, the scattering of the cylindrical canyon can always be expressed by the sum of wave function series. In order to describe the scattering effects intuitively, according to Eq. (25), introduce the dynamic stress concentration factor $\tau_{\theta z} *$ on the edge of the circular canyon. It can be used to characterize the total wave field near the cylinder, i.e., the near field solution. Because of the existence of horizontal boundary $B_{H}$ and vertical boundary $B_{V}$, if the scattering effect is not considered, $\tau_{\theta z} *$ will be no more than 4 .

$$
\tau_{\theta z}^{*}=\frac{\left|\tau_{\theta z}^{s}(z)+\tau_{\theta z}^{p}(z)\right|}{W k \mu}
$$

\section{Asymptotic and far field solutions.}

Once the scattering wave of the cylinder is formed, it will spread outward. In order to quantitatively study the propagation of scattering wave, introduce the asymptotic behavior of the first kind of Hankel function at the infinity point, Eq. (26); the displacement field of scattering wave $w_{s}$ is expressed as Eq. (27). Here, $F(\theta)$ is the far field displacement mode of scattered wave $w_{s}$, in accordance with the Graf addition formula (28), Eq. (29) is defined. The convergence of the series (28) which is given by the Graf addition formula depends on the parameter $2 k d$, and when it is given, the finite terms of series (7) and (28) can be used to ensure that the series (29) has sufficient accuracy.

The far field displacement model $F(\theta)$ of the scattering wave $w_{s}$ represents the directionality of cylindrical waves propagating from the center of the canyon to the point of infinity. The directionality of the cylindrical scattering wave is a combination of the near field scattering 
effect and the asymptotic behavior of the displacement wave at infinity, and this is called far field solution.

$$
\begin{aligned}
& H_{n}^{(1)}(\bullet) \stackrel{\bullet \rightarrow \infty}{\longrightarrow} \sqrt{\frac{2}{\pi} \bullet} \exp \left[i\left(\bullet-\frac{1+2 n}{4} \pi\right)\right] \\
& w_{s}(z) \stackrel{|z| \rightarrow \infty}{\longrightarrow} \sqrt{\frac{2}{\pi k r}} \exp \left[i\left(k r-\frac{\pi}{4}\right)\right] F(\theta) \\
& \left.D^{\prime}\left(z^{\prime}\right)=(-1)^{n} H_{n}^{(1)}\left(k\left|z^{\prime}\right|\right)\left(\frac{z^{\prime}}{\left|z^{\prime}\right|}\right)^{-n}=\sum_{m=-\infty}^{+\infty}(-1)^{n+m} H_{n+m}^{(1)}(k \mid z)\right) J_{m}(2 k d)\left(\frac{z}{|z|}\right)^{-(n+m)} \\
& F(\theta)=\sum_{n=-\infty}^{+\infty} A_{n} e^{i n\left(\theta-\frac{\pi}{2}\right)}+\sum_{n=-\infty}^{+\infty} \sum_{m=-\infty}^{+\infty}(-1)^{n+m} A_{n} J_{m}(2 k d) e^{-i(n+m)\left(\theta+\frac{\pi}{2}\right)}
\end{aligned}
$$

\section{Conclusions}

According to wave function expansion method and mirror image method, the anti plane scattering of steady state SH-plane wave by semi cylindrical canyon in a quarter space is studied. The dynamic stress concentration factor which represent the near field solution and the displacement mode which represent the far field solution are obtained. The result shows:(1) The value and distribution of the dynamic stress concentration caused by scattering wave of the canyon depend on the standing wave which formed by plane waves in the quarter space. (2) The far field displacement mode of the scattering wave by the canyon depends on the product of its distance from the vertical boundary and the wave number of the incident wave, and also related to the incidence angle. These conclusions have certain value in stress analysis and material inspection.

\section{References :}

[1] Trifunac M.D., Scattering of plane SH waves by a semi-cylindrical canyon, Earth Engineering and Structural Dynamics, 1 (1973) 267-281

[2] Pao Y. H. and Mow C. C.: Diffraction of Elastic Waves and Dynamic Stress Concentrations, Crane and Russak ,New York, (1973)

[3] Qiu Fa Qiang, Wang Hui Wen and Wang Xue, The interaction between non isosceles triangular structure and foundation under SH wave incidence, Journal of Harbin Institute of Technology, 42 (2010) 634-638

[4] Lee V. W., On deformation near circular underground cavity subjected to incident plane SH waves, Proceedings of the application of computer methods in engineering conference, Los Angeles (1977) 951-652

[5] Liu Hongwei, Liu Diankui, Far field solution of SH-wave scattered by interface circualr hole, Acta Mechanica Solida Sinica, 20 (1999) 349-355

[6] Liu Diankui, Wang Guoqing, Antiplane SH-deformation of a semi-cylindrical hill above a subsurface cavity, Acta Mechanica Sinica, 3(2006) 209-218

[7] Liang Jianwen, Zhang Yushan, Gu Xiaolu, Lee V. W., Scattering of plane SH waves by a circular-arc layered canyon , Journal of Vibration Engineering, 16 (2003) 158-165

[8] Liu Zhongxian, Liang Jianwen, Analytic solution for diffraction of plane SH waves by a circular alluvial valley in wedge-shaped space, Journal of Tianjin University, 43 (2010) 573-582

[9] Chen Tianyu, Wang Hui. Dynamic analysis of upright incidence of SH-wave at semi-cylindrical interface with a circular lining structure, Journal of Tianjin University 39 
(2006) 1305-1309

[10] Shi Yong, Qi Hui, Yang Zailin, Scattering of SH-wave by circular cavity in right-angle plane and seismic ground motion, Chinese Journal of Applied Mechanics, 25 (2008) 392-398

[11]Qi Hui, Shi Yong, Li Hong Liang and Zhao Jia Xi, Dynamic analysis of the circular hole near the interface between two phases in a vertical half space with SH wave incidence, Explosion and Shock Waves, 29 (2009)73-79

[12]Qi H., Shi Y. and Liu D. K., Interaction of a circular cavity and a beeline crack in right-angle plane impacted by SH-wave, Journal of Harbin Institute of Technology 16 (2009) 548-553

[13]Qi H., Yang J., Shi Y., Tian J. Y., Dynamic analysis for circular inclusion near interfacial crack impacted by SH wave in half space, Journal of Mechanics 28 (2012) 143-151

[14]Qi H., Yang J., Dynamic analysis for circular inclusions of arbitrary positions near interfacial crack impacted by SH-wave in half-space, European Journal of Mechanics - A/Solids 36(2012) $18-24$

[15]Qi H., Yang J., Shi Y., Scattering of SH-wave by cylindrical inclusion near interface in bi-material half space, Journal of Mechanics 27 (2011) 37-45 